\title{
EFEITO DO VALOR REFORCADOR DO ALIMENTO EM DIFERENTES TEMPOS DE PRIVAÇÃO ALIMENTAR
}

\section{Vivian Costa Resende Cunha}

Nutricionista. Doutoranda em Psicologia pela Pontifícia Universidade Católica de Goiás, Brasil.

\section{Sônia Maria Mello Neves}

Docente Titular do curso de Psicologia, Escola de Ciências Sociais e da Saúde da Pontifícia Universidade Católica de Goiás. Doutora em Psicologia Experimental, Brasil.

\section{Renata Mendes de Souza} Laís Melo Giglio

Graduandas em Psicologia pela Pontifícia Universidade Católica de Goiás, Brasil.

\section{Mariana Carvalho Meira} Kássia Santos Fernandes

Graduandas em Nutrição pela Pontifícia Universidade Católica de Goiás, Brasil.
RESUMO: Avaliar o valor reforçador do alimento sob diferentes tempos de privação; até 4 horas (grupo tempo 1 - GT1) e mais de 4 horas (grupo tempo 2 - GT2), em mulheres e homens eutróficos e com excesso de peso, classificados pelo índice de massa corporal (IMC). Foram ouvidos 258 adultos, sendo 126 homens e 132 mulheres (18 50 anos), foram divididos em GT1 ou GT2. Após classificarem a fome pela escala analógica visual $100 \mathrm{~mm}$, realizaram um jogo desenvolvido para este estudo (software Food or Fun 1.0), onde deveriam trabalhar para ganhar pontos em alimento ou lazer. Na amostra geral o relato de fome diferiu estatisticamente $(\mathrm{p}<0,001)$ entre GT1 $(42,4 \pm 25,9)$ e GT2 $(60,2 \pm 22,4)$ assim como a pontuação em alimentos $(\mathrm{GT} 1=26,7$ $\pm 23,5 ;$ GT2 $=35,2 \pm 23,4 ; p<0,001)$, demonstrando uma tendência diretamente proporcional ao tempo de privação em ambos os sexos. Uma correlação negativa significativa foi encontrada entre IMC e pontos em alimentos para a amostra geral $(r=-0,16 ; p=0,013)$ e do sexo feminino $(r=-0,18 ; p=0,040)$. O valor reforçador do alimento aumentou concomitantemente com o tempo de privação e IMC apresentou uma correlação inversa à pontuação em alimentos.

PALAVRAS-CHAVE: Motivação; Comportamento alimentar; Peso corporal; Privação de alimentos.

\section{EFFECT OF FOOD REINFORCEMENT VALUE AT DIFFE- RENT DEPRIVATION PERIODS}

\begin{abstract}
Current paper evaluates the reinforcing value of food at different deprivation periods: up to $4 \mathrm{~h}$ (Group Time 1 - GT1) and more than $4 \mathrm{~h}$ (Group Time 2 - GT2), in eutrophic females and males, weight excess, classified by Body Mass Index (BMI). Divided into GT1 and GT 2, 258 adults, 126 males and 132 females, age bracket 18 -50 years, were interviewed. After the classification of fasting by the $100 \mathrm{~m}$ visual analogical scale, a game was developed for current study (software Food or Fun 1.0), in which they had to work for scores in food or fun. Within the general sample, there was a statistical difference in fasting $(\mathrm{p}<0.001)$ between GT1 $(42.4 \pm 25.9)$ and GT2 (60.2 \pm 22.4) and in feeding scores $(\mathrm{GT} 1=26.7 \pm 23.5$; GT2 $=35.2 \pm 23.4$; $\mathrm{p}<0.001$ ), with a proportional trend to deprivation time in both genders. A significant negative co-relationship was detected between BMI and feeding scores for general sample $(r=-0.16 ; p=0.013)$ and females $(r=-0.18 ; p=0.040)$. Reinforcing value of feeding increased concomitantly with privation period and BMI had an inverse co-relationship to feeding scores.
\end{abstract}

KEY WORDS: Motivation; Feeding behavior; Body weight; Lack of food. 


\section{INTRODUÇÃO}

A obesidade tem sido considerada a mais importante doença nutricional nos países com renda baixa, média e alta, pelo aumento de sua incidência, considerada uma pandemia mundial ${ }^{1-4}$. A privação alimentar (redução calórica) e a restrição alimentar (acesso limitado a alguns alimentos) têm sido componentes centrais no tratamento nutricional da obesidade e do sobrepeso5. Tal abordagem nutricional, utilizando dietas de cunho privativo e restritivo embora seja disseminada e recorrente desde a antiguidade, não tem demonstrado sucesso na perda de peso em longo prazo, com a grande maioria das pessoas recuperando todo peso perdido em pouco tempo ${ }^{6-9}$.

$\mathrm{Na}$ perspectiva, Behaviorista Radical, o estudo do comportamento alimentar deve considerar ser inicialmente uma resposta respondente uma vez que todo organismo necessita do alimento para o seu crescimento e manutenção e também um comportamento operante, por gerar efeitos no ambiente e ser afetado pelas modificações que produz (e.g., ganho de peso, alívio das chateações etc. $)^{10,11}$. Uma vez que cada comportamento é selecionado pelas consequências que produzem, logo as consequências que aumentam a probabilidade de uma determinada resposta voltar a ocorrer, são denominadas de reforço ${ }^{12}$.

Neste contexto, Epstein e colaboradores ${ }^{5,13,14}$ desenvolveram uma medida de valor reforçador do alimento, a qual se baseou em pesquisas relacionadas que utilizaram uma abordagem de medição similar no contexto de abuso de drogas. O valor reforçador do alimento pode ser definido em quantas respostas são necessárias/ feitas para se obter/escolher o alimento ${ }^{14}$. Um indivíduo motivado para o alimento/comer implica dizer que entre tantas escolhas para serem feitas em um contexto de alternativas, ele escolheu a opção alimento ${ }^{13}$.

Estudiosos $^{5}$ avaliaram o efeito da privação alimentar (calórica) e restrição alimentar (acesso limitado a alguns alimentos) isoladas ou combinadas, sobre o valor reforçador do alimento e o consumo alimentar. $\mathrm{O}$ estudo concluiu que nas participantes com peso normal (IMC aproximadamente $21,5 \mathrm{~kg} / \mathrm{m}^{2}$ ), saudáveis e comedoras não restritas, a situação de privação alimentar (de em média 17 horas), levou a um maior relato de fome e aumento do valor reforçador do alimento, porém, a restrição alimentar a curto prazo (15 minutos) não levou ao aumento do valor reforçador do alimento.

Drobes et al. ${ }^{15}$ avaliaram o efeito da privação alimentar de 0,6 e 24 horas nas reações emocionais e consumo alimentar e encontraram uma correlação positiva entre o tempo de privação e o consumo alimentar e observaram que os desejos alimentares aumentavam quanto maior o tempo de privação alimentar. Embora este estudo não tenha usado a medida de valor reforçador do alimento para acessar a motivação para o alimento, os pesquisadores concluíram que o desejo e o consumo estavam aumentados após os diferentes tempos de privação ${ }^{15}$.

Diversas variáveis influenciam diretamente o valor reforçador do alimento. Em uma situação de escolha, o valor reforçador do alimento será influenciado pelo valor reforçador de alternativas, em esquemas concorrentes. O índice de massa corporal (IMC) também parece ser uma variável que influencia diretamente o valor reforçador do alimento. Indivíduos obesos tendem a achar o alimento mais reforçador, que indivíduos não obesos ${ }^{16} \mathrm{e}$ diferentes IMC respondem de maneira diferente ao valor reforçador dos alimentos de elevada palatabilidade ${ }^{17,18}$.

A variável sexo também parece influenciar as propriedades de reforço de algumas substâncias. Diferenças na autoadministração de drogas e na sensibilidade à droga são relatadas na literatura, tendo o sexo como variável. Estas diferenças são atribuídas, pelo menos em parte, às diferenças hormonais e por este motivo o ciclo menstrual da mulher parece influenciar nesta sensibilidade ${ }^{19,20}$.

Considerando que os estudos têm avaliado o efeito de privação alimentar de longo prazo (17 horas) no valor reforçador do alimento, até o momento não foram encontrados estudos que avaliassem diferentes tempos de privação no valor reforçador do alimento, portanto, este estudo é o pioneiro em realizar esta análise. A hipótese a ser testada é de que quanto maior o tempo de privação, maior o valor reforçador do alimento e que os indivíduos com excesso de peso tenham motivação maior para o alimento comparado aos eutróficos (peso adequado para altura). Desta forma, o objetivo deste estudo consiste em avaliar o efeito de diferentes tempos de privação alimentar (variável independente) sobre o valor 
reforçador do alimento, ou seja, escolha por lazer ou alimento (variável dependente) e relato de fome (variável dependente) em mulheres e homens eutróficos e com excesso de peso (variáveis moderadoras).

\section{MÉTODO}

Participaram do estudo 258 indivíduos, 126 homens (63 eutróficos e 63 com excesso de peso) e 132 mulheres (73 eutróficas e 59 com excesso de peso), com idade entre 18 e 50 anos $(22,1 \pm 5,0)$, embora a maior parte dos participantes (91\%) tenha idade entre 18 e 28 anos.

Os participantes foram recrutados nas cantinas da área III e IV da Pontifícia Universidade Católica de Goiás e o experimento foi realizado no Laboratório de Análise Experimental do Comportamento (LAEC), entre os meses de fevereiro e junho de 2017.

Para a análise do relato verbal de fome utilizou-se a escala visual analógica $100 \mathrm{~mm}$ de fome (escalas de $100 \mathrm{~mm}$ utilizada para medida de fome que variou entre "com nada de fome" - 0 - e "extremamente com fome" 100). A anamnese utilizada continha questóes referentes a nome, sexo, data de nascimento, peso, altura e tempo de privação.

Para realização da coleta de dados, o software Food or Fun1. $0^{21}$ foi desenvolvido com a linguagem pascal na plataforma Delphi 7. O software consiste em um jogo onde os participantes poderiam ganhar pontos para alimentos ou atividades de lazer. Antes de iniciar o jogo, a seguinte instrução foi apresentada na tela do computador: "Neste jogo você terá que escolher entre duas opções: alimento ou lazer. Ao escolher uma destas alternativas, você ganhará pontos. Esses pontos serão trocados, ao final do jogo, por alimento ou atividade de lazer. Escolha a opção que te dará pontos para obter o que mais deseja". Durante o jogo os participantes escolheram entre duas alternativas (alimento ou atividade de lazer - esquemas concorrentes) que foram apresentadas em uma tela, no qual o toque/resposta dado a cada uma destas alternativas produzia pontos que eram computados e apresentados na tela abaixo de cada alternativa (Figura 1). Os denominados esquemas concorrentes se referem a duas fontes de reforço disponíveis ao mesmo tempo como, por exemplo, comer ou assistir televisão e são os mais presentes e importantes esquemas presentes no nosso cotidiano ${ }^{22,23}$.

A liberação dos pontos foi programada em diferentes esquemas de reforçamento de razão variável. Para a alternativa correspondente à atividade de lazer, o esquema de liberação de pontos foi a razão variável 2 (VR2) e para a alternativa correspondente ao alimento, esteve programado um esquema de razão variável encadeada (VR2, VR4, VR6, VR8, VR10). Em um esquema de VR a apresentação de um reforçador depende da emissão de um número médio de respostas, independentemente da passagem do tempo. Para a liberação de pontos para lazer, para emissão do ponto eram necessárias, em média, duas respostas. Para a alternativa alimento, o número de respostas para emissão do ponto variou de 2 a 10, com o passar do jogo, a fim de medir o valor reforçador desta alternativa. Neste esquema as taxas de respostas são maiores, uma vez que o indivíduo não consegue discriminar o número de respostas necessárias para obter o reforço e ao aumentar a frequência do responder, aumenta a probabilidade de suas respostas serem reforçadas ${ }^{22}$.

Os participantes trocaram os pontos adquiridos durante a sessão experimental, no software, por alimentos ou atividades de lazer e brindes como fichas de sinuca, ingressos de cinema, vale tapioca e vale salgado. 


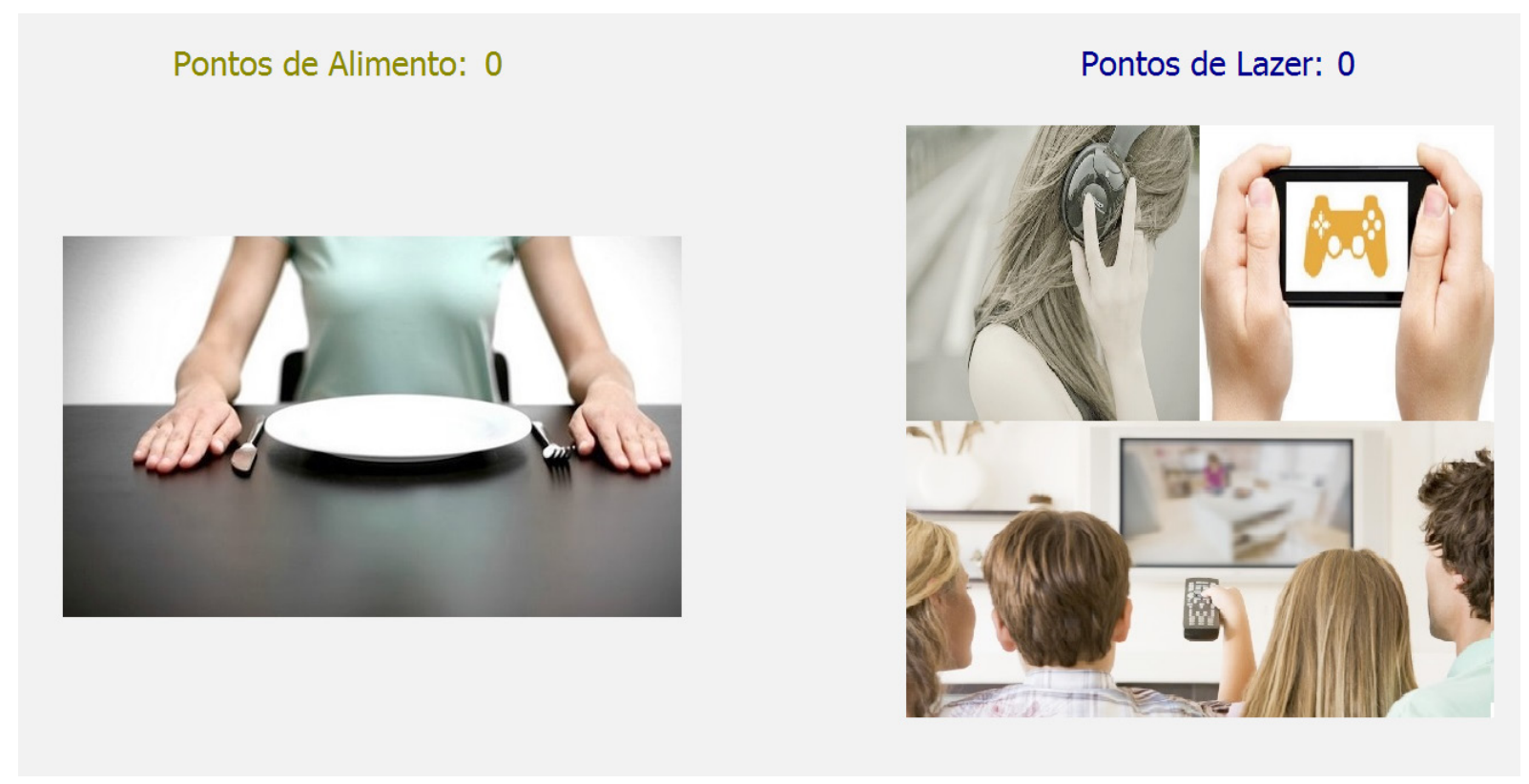

Figura 1. Tela inicial do software onde são apresentados os reforçadores e a pontuação acumulada durante o jogo

Este estudo foi aprovado pelo Comitê de Ética da Pontifícia Universidade Católica de Goiás (CEP-PUC/GO) sob o protocolo número 54025616.3.0000.0037, parecer 1.548.538.

Em um primeiro momento, fez-se o recrutamento dos participantes nas cantinas das áreas III e IV da PUC-GO. Após explicação da pesquisa, os participantes que concordaram em participar assinaram o Termo de Consentimento Livre e Esclarecido (TCLE). Posteriormente, foi solicitado aos participantes que informassem o nome, data de nascimento e em seguida foram encaminhados ao LAEC onde preencheram a escala visual analógica 100 $\mathrm{mm}$ de fome.

Após avaliar o nível de fome inicial, os participantes foram questionados há quanto tempo tinham realizado a última refeição, ou seja, o tempo que estavam privados de comida (qualquer quantidade, alimento ou valor nutricional) até o momento da pesquisa. O tempo de privação alimentar foi categorizado em ois grupos: até 4 horas privado (GT1) ou mais de 4 horas (GT2). Em seguida, os participantes foram conduzidos até uma sala onde foram instruídos de como utilizar o jogo de computador desenvolvido para este estudo (software - Food or Fun 1.0).

Após realizarem o jogo, os participantes foram questionados sobre o peso e altura e ao final, aqueles que ganharam mais pontos para alimento receberam um brinde de sua escolha, referente a alimento, que consistia em vale tapioca ou vale salgado. Os participantes que ganharam mais pontos para lazer escolheram entre vale cinema e vale sinuca, como brindes disponíveis para atividade de lazer.

O programa Microsoft ${ }^{\circledR}$ Excel 2007 foi usado para tabulação dos dados e para realização da análise estatística descritiva. A análise estatística inferencial foi realizada pelo programa SPSS ${ }^{\circledR}$ for Windows ${ }^{\circledR}$, versão 16.0. Para avaliar a influência entre o tempo de privação alimentar (variável independente) sobre o valor reforçador do alimento, ou seja, pontos em lazer ou alimento (variável dependente) e relato de fome (variável dependente) em mulheres e homens eutróficos e com excesso de peso (variáveis moderadoras) foram utilizados os testes Qui-Quadrado e Mann-Whitney para dados não paramétricos, confirmados pelo teste Kolmogorov-Smirnov. A análise de correlação foi realizada para medir a intensidade da influência entre as variáveis (IMC, fome, pontos em alimentos ou lazer, sexo e tempo de privação). Para a análise de correlação foi utilizado o teste Pearson, pois as variáveis eram escalares. Os valores de coeficientes de correlação encontrados foram classificados seguindo a metodologia de Hopkins ${ }^{24}$, onde: $0>\mathrm{r}>0,01$ - muito baixa; $0,1>\mathrm{r}>0,3$ - baixa; $0,3>\mathrm{r}>0,5$ - moderada; $0,5>\mathrm{r}>0,7$ - alta; $0,7>\mathrm{r}>0,9-$ muito alta; $0,9>$ $r>1,0-$ quase perfeita. Adotou-se um $p<0,05$ para todas as análises conduzidas no presente estudo. 


\section{RESULTADOS}

Não foi encontrada diferença significativa para idade, peso, altura e IMC entre os dois grupos (GT1 e GT2), indicando amostras homogêneas. O relato de fome foi diferente, significativamente, entre os dois grupos, uma vez que o grupo que estava até 4 horas privado teve uma fome média de 42,4 \pm 25,9 enquanto os participantes que estavam privados por mais de 4 horas apresentaram fome média de 60,2 $\pm 22,4(\mathrm{p}<0,01)$ (Tabela 1).

Tabela 1. Média e desvio-padrão do IMC, relato de fome, pontos em alimento e pontos em lazer em relação ao tempo de privação (GT1 e GT2) da amostra geral

\begin{tabular}{cccc}
\hline & Média & DP & $\boldsymbol{p}$ \\
\hline IMC & & & \\
GT1 & 25,8 & 5,2 & \\
GT2 & 25,4 & 4,7 & 0,670 \\
\hline \multicolumn{2}{l}{ Relato de fome } & & \\
\hline GT1 & 42,4 & 25,9 & \\
GT2 & 60,2 & 22,4 & $<0,001^{*}$ \\
\hline \multicolumn{2}{l}{ Pontos em alimento } & & \\
\hline GT1 & 26,7 & 23,5 & \\
GT2 & 35,2 & 23,4 & $0,001^{*}$ \\
\hline Pontos em lazer & & \\
\hline GT1 & 48,3 & 23,5 & \\
GT2 & 39,8 & 23,4 & $0,001^{*}$ \\
\hline
\end{tabular}

Nota: $\mathrm{DP}=$ desvio-padrão; IMC = índice de massa corporal; GT1 = grupo até 4 h de privação; GT2 = grupo com mais de 4 h de privação; $*=p<0,05$.

A diferença significativa para a fome foi encontrada tanto na amostra geral, quanto para os participantes do sexo feminino e masculino. Houve diferença significativa para respostas em alternativas que sinalizavam acesso a alimentos, denominado pontos em alimentos, entre os dois grupos (GT1 e GT2) $(p=0,001)$. Os pontos em alimentos apresentaram tendência a aumentar à medida que aumentava o tempo de privação $(p=0,001)$, enquanto foi observada diminuição na pontuação de lazer, quanto maior o tempo de privação, na amostra geral (Tabela 1).

Quando a amostra foi separada por sexo, também foi encontrada a mesma tendência, com diferença significativa na pontuação para alimentos e lazer. A média de pontos em alimentos para a amostra geral no GT1 foi de 26,7 $\pm 23,5$ e no GT2 de 35,2 $\pm 23,4$ e de pontos para lazer no grupo GT1 foi de 48,3 $\pm 23,5$ e no GT2 de 39,8 $\pm 23,4$. Quando dividido por sexo, o sexo masculino no grupo GT1 pontuou para alimentos uma média de 25,7 $\pm 23,5$ pontos e o grupo GT2, 33,7 $\pm 23,9$. Já os pontos em lazer do sexo masculino corresponderam a $49,3 \pm$ 23,5 para GT1 e 41,3 $\pm 23,9$ para GT2. Quando analisado o sexo feminino, o grupo GT1 pontuou 27,6 $\pm 23,7$ pontos para alimento e $47,3 \pm 23,7$ pontos para lazer, enquanto o grupo GT2 obteve $36,6 \pm 23,0$ pontos em alimento e 38,4 $\pm 23,0$ pontos em lazer (Tabelas 2 e 3).

Tabela 2. Média e desvio-padrão do IMC, relato de fome, pontos em alimento e pontos em lazer em relação ao tempo de privação (GT1 e GT2) da amostra do sexo masculino

\begin{tabular}{cccc}
\hline & Média & DP & $\boldsymbol{p}$ \\
\hline IMC & & & \\
GT1 & 25,9 & 4,7 & \\
GT2 & 25,3 & 4,3 & 0,493 \\
\hline Relato de fome & & & \\
\hline GT1 & 42,3 & 23,5 & \\
GT2 & 56,7 & 21,4 & $0,001^{* *}$ \\
\hline Pontos em alimento & & & \\
\hline GT1 & 25,7 & 23,5 & \\
GT2 & 33,7 & 23,9 & $0,038^{*}$ \\
\hline Pontos em lazer & & & \\
\hline GT1 & 49,3 & 23,5 & \\
GT2 & 41,3 & 23,9 & $0,040^{*}$ \\
\hline
\end{tabular}

Nota: DP = desvio-padrão; IMC = índice de massa corporal; GT1 = grupo até 4h de privação; GT2 = grupo com mais de 4 h de privação; ** $=p<0,001 ; *=p<0,05$ 
Tabela 3. Média e desvio-padrão do IMC, relato de fome, pontos em alimento e pontos em lazer em relação ao tempo de privação (GT1 e GT2) da amostra do sexo feminino

\begin{tabular}{cccc}
\hline & Média & DP & $\boldsymbol{p}$ \\
\hline IMC & & & \\
GT1 & 25,8 & 5,7 & \\
GT2 & 25,5 & 5,0 & 0,857
\end{tabular}

\begin{tabular}{cccc}
\hline Relato de fome & & & \\
\hline GT1 & 42,4 & 28,2 & \\
GT2 & 63,5 & 23,1 & $<0,001^{* *}$ \\
\hline
\end{tabular}

\begin{tabular}{cccc}
\hline Pontos em alimento & & & \\
\hline GT1 & 27,6 & 23,7 & \\
GT2 & 36,6 & 23,0 & $0,014^{*}$ \\
\hline
\end{tabular}

\begin{tabular}{|c|c|c|c|}
\hline \multicolumn{4}{|c|}{ Pontos em lazer } \\
\hline GT1 & 47,3 & 23,7 & \\
\hline GT2 & 38,4 & 23,0 & $0,014^{*}$ \\
\hline
\end{tabular}

Nota: DP $=$ desvio-padrão; IMC $=$ índice de massa corporal; GT1 $=$ grupo até 4h de privação; GT2 = grupo com mais de 4h de privação; ** $=p<0,001 ; *=p<0,05$

A partir da análise de correlação de Pearson, foi encontrada uma correlação negativa baixa, embora significativa, entre o IMC e a pontuação em alimentos para a amostra geral e do sexo feminino; porém, a correlação negativa não foi significativa para o sexo masculino. A fome apresentou uma correlação positiva moderada e significativa com a pontuação em alimentos e negativa e significativa com a pontuação em lazer, tanto para a amostra geral quanto para a subdivisão do sexo masculino e feminino $(p<0,001)$. Quando analisada a correlação nos diferentes grupos, observou-se que embora as variáveis IMC e pontuação em lazer tenham apresentado correlação positiva baixa, ela foi significativa apenas para a amostra geral e sexo feminino que estavam até 4 h privada de alimento (GT1), enquanto a fome manteve a correlação positiva com a pontuação de alimentos e em todas as estratificações (amostra geral, sexo masculino, sexo feminino e diferentes tempos de privação) ela foi significativa. A correlação entre fome e pontos em alimento e lazer foi significativa para ambos os grupos na amostra geral e quando separada por sexo, sendo classificada como moderada no GT1 e baixa no GT2 (Tabelas 4 e 5).
Tabela 4. Correlação do IMC e relato de fome com a pontuação em alimentos (PA) e lazer (PL) na amostra geral, no sexo masculino e sexo feminino

\begin{tabular}{ccccc}
\hline & Variáveis & r & $\begin{array}{c}\text { Classificação } \\
\text { das correla- } \\
\text { ções }\end{array}$ & $\boldsymbol{p}$ \\
\hline $\begin{array}{c}\text { Amostra } \\
\text { geral }\end{array}$ & IMC e PA & $-0,18$ & Baixa & $0,004^{*}$ \\
& IMC e PL & 0,18 & Baixa & $0,004^{*}$ \\
& Fome e PA & 0,32 & Moderada & $<0,001^{* *}$ \\
& Fome e PL & $-0,32$ & Moderada & $<0,001^{* *}$ \\
$\begin{array}{c}\text { Sexo } \\
\text { masculino }\end{array}$ & IMC e PA & $-0,17$ & Baixa & 0,064 \\
& IMC e PL & 0,17 & Baixa & 0,063 \\
& Fome e PA & 0,31 & Moderada & $<0,001^{* *}$ \\
& Fome e PL & $-0,31$ & Moderada & $<0,001^{* *}$ \\
$\begin{array}{c}\text { Sexo } \\
\text { feminino }\end{array}$ & IMC e PA & $-0,19$ & Baixa & $0,030^{*}$ \\
& IMC e PL & 0,19 & Baixa & $0,030^{*}$ \\
& Fome e PA & 0,33 & Moderada & $<0,001^{* *}$ \\
& Fome e PL & $-0,33$ & Moderada & $<0,001^{* *}$ \\
\hline
\end{tabular}

Nota: $\mathrm{DP}=$ desvio-padrão; IMC = índice de massa corporal; GT1 $=$ grupo até 4h de privação; GT2 = grupo com mais de $4 \mathrm{~h}$ de privação; ** $=p<0,001 ; *=p<0,05$; Correlação de Pearson. Seguiu-se a classificação proposta por Hopkins ${ }^{23}$ para classificação das correlações. 
Tabela 5. Correlação do IMC e relato de fome com a pontuação em alimentos (PA) e lazer (PL) na amostra geral, sexo masculino e sexo feminino, nos diferentes tempos de privação

\begin{tabular}{|c|c|c|c|c|c|c|c|}
\hline & \multirow[b]{2}{*}{ Variáveis } & \multicolumn{3}{|c|}{ GT1 } & \multicolumn{3}{|c|}{ GT2 } \\
\hline & & $\mathbf{r}$ & $\begin{array}{l}\text { Classificação } \\
\text { das } \\
\text { correlações }\end{array}$ & $p$ & $\mathbf{r}$ & $\begin{array}{c}\text { Classificação } \\
\text { das } \\
\text { correlações }\end{array}$ & $p$ \\
\hline \multirow[t]{4}{*}{ Amostra geral } & IMC e PA & $-0,17$ & Baixa & $0,004^{*}$ & $-0,12$ & Baixa & 0,181 \\
\hline & IMC e PL & 0,17 & Baixa & $0,004^{*}$ & 0,12 & Baixa & 0,181 \\
\hline & Fome e PA & 0,32 & Moderada & $<0,001 * *$ & 0,29 & Baixa & $0,002 *$ \\
\hline & Fome e PL & $-0,32$ & Moderada & $<0,001^{* *}$ & $-0,29$ & Baixa & $0,002^{*}$ \\
\hline \multirow[t]{4}{*}{ Sexo masculino } & IMC e PA & $-0,17$ & Baixa & 0,064 & $-0,08$ & Muito baixa & 0,533 \\
\hline & IMC e PL & 0,17 & Baixa & 0,063 & 0,08 & Muito baixa & 0,533 \\
\hline & Fome e PA & 0,31 & Moderada & $<0,001^{* *}$ & 0,28 & Baixa & $0,032 *$ \\
\hline & Fome e PL & $-0,31$ & Moderada & $<0,001^{* *}$ & $-0,28$ & Baixa & $0,032 *$ \\
\hline \multirow[t]{4}{*}{ Sexo feminino } & IMC e PA & $-0,19$ & Baixa & $0,030^{*}$ & $-0,16$ & Baixa & 0,215 \\
\hline & IMC e PL & 0,19 & Baixa & $0,030^{*}$ & 0,16 & Baixa & 0,215 \\
\hline & Fome e PA & 0,33 & Moderada & $<0,001^{* *}$ & 0,28 & Baixa & $0,030^{*}$ \\
\hline & Fome e PL & $-0,33$ & Moderada & $<0,001^{* *}$ & $-0,28$ & Baixa & $0,030^{*}$ \\
\hline
\end{tabular}

Nota: $\mathrm{DP}=$ desvio-padrão; IMC = índice de massa corporal; GT1 = grupo até 4h de privação; GT2 = grupo com mais de 4h de privação; $* *=p<0,001 ; *=p<0,05$; Correlação de Pearson. Seguiu-se a classificação proposta por Hopkins ${ }^{23}$ para classificação das correlações.

\section{DISCUSSÃO}

Este estudo foi pioneiro em analisar o efeito de diferentes tempos de privação no valor reforçador do alimento, assim como esta relação em indivíduos de ambos os sexos e diferentes IMC. Os resultados encontrados demonstraram que o tempo de privação influenciou significativamente o valor reforçador do alimento, para ambos os sexos, uma vez que o grupo privado de até 4 horas pontuou menos para o alimento do que o grupo que estava há mais de 4 horas privado.

Diversos estudos corroboram com o resultado encontrado, de que a privação alimentar aumenta a recompensa e motivação para o alimento $0^{5,7,15,25,26}$. Além de aumentar a motivação para o alimento, estudos têm demonstrado que o valor reforçador do alimento também parece estar relacionado com a impulsividade no consumo de alimentos ${ }^{27,28}$, o que pode explicar a ineficácia das dietas (com privação alimentar) para a perda de peso. Na recente revisão de literatura ${ }^{29}$, os autores concluem que há evidências consistentes de que os adultos obesos apresentam maior valor reforçador do alimento.

Além das relações de dependência encontradas na literatura entre mudanças ambientais e comportamento, ou seja, horas de privação de alimento e aumento na frequência de resposta que levam ao alimento, indicando um aumento no valor reforçador do alimento, outras alterações metabólicas também têm sido associadas à privação alimentar como a sua relação com o sistema dopaminérgico. $\mathrm{O}$ aumento do valor reforçador tem sido relacionado à atividade do sistema dopaminérgico, uma vez que este sistema está relacionado com o prazer oferecido por recompensas naturais, como os alimentos ${ }^{30,31}$. A privação alimentar crônica tem sido associada ao aumento do valor de recompensa/reforçador de alimentos e consumo alimentar pelo aumento da disponibilidade ou diminuição da receptação da dopamina ${ }^{32}$. Em indivíduos como maior IMC, o sistema dopaminérgico parece estar alterado, apresentando deficiência no sistema de recompensa, denominada de "síndrome da deficiência 
de recompensa", favorecendo maior apetite e consumo alimentar ${ }^{31,33}$.

O conhecimento sobre a influência da privação alimentar (componente do tratamento para a perda de peso) na motivação para o alimento/comer e, consequentemente, no consumo alimentar, é de fundamental importância para a prática clínica de profissionais nutricionistas, com vista a auxiliar no manejo nutricional para a perda de peso sustentável em longo prazo. Mais estudos são necessários para investigar os fatores e processos envolvidos nas consequências negativas da privação alimentar para o consumo alimentar e/ou ganho de peso, de forma a contribuir para a aplicação clínica dos profissionais.

Embora o resultado tenha demonstrado uma diferença significativa nos pontos em alimentos entre os grupos, quando analisada a média dos pontos obtidos, observa-se que tanto a amostra geral quanto avaliada por sexo feminino e masculino, o grupo GT1 obteve uma média maior para a pontuação de lazer. Uma possível explicação para este dado se refere aos reforçadores (brindes) que foram entregues aos participantes após o término do experimento, que eram correspondentes à pontuação no software. Os brindes de lazer se referiam a vale cinema ou vale sinuca, que poderiam ser grandes reforçadores para o público em questão (prioritariamente universitários) $)^{34}$.

O tempo de privação alimentar também influenciou o relato de fome dos participantes. Observou-se aumento significativo no relato de fome proporcionalmente ao tempo de privação alimentar, corroborando com dados da literatura ${ }^{35,36,37}$, que indicam que períodos de privação alimentar estão associados ao aumento da percepção de fome.

A grande maioria dos estudos sugere que a tentativa de descrever verbalmente eventos não verbais pode gerar relatos que não fazem jus à experiência original, uma vez que para os indivíduos se concentrarem em informações relevantes verbalmente, pode fazer com que eles percam o contato com seus verdadeiros sentimentos/ sensações ${ }^{38,39}$.Corroborando com esta premissa, Creswell et $\mathrm{al}^{40}{ }^{40}$ encontraram que são mais confiáveis ferramentas de medidas não verbais (como o dinamômetro) para medir a fome, uma vez que o relato verbal não foi congruente com o comportamento alimentar. No presente estudo não foram utilizadas medidas não verbais para medir a fome, apenas o relato, entretanto, refutando alguns resultados encontrados previamente ${ }^{38-40} \mathrm{o}$ relato verbal de fome foi congruente com o comportamento alimentar, uma vez que foi encontrada uma correlação positiva significativa para o relato de fome e a pontuação em alimentos para toda a amostra. Tal correspondência encontrada entre a fome e a pontuação em alimentos pode ser explicada pela regulação homeostática do organismo, como mecanismo de sobrevivência dos seres humanos, tornando a espécie especialmente sensível aos sinais de fome ${ }^{41}$.

Foi encontrada uma relação inversa significativa entre IMC e pontuação em alimentos, ou seja, quanto maior o IMC dos participantes, menor a pontuação em alimentos. Este resultado refuta alguns achados na literatura que encontraram uma relação positiva entre o peso corporal e o valor reforçador do alimento ${ }^{18,42}$. Embora neste estudo não tenha sido avaliada a presença de comportamento alimentar inadequado (ou comer transtornado) ou a prática de fazer dieta, estas variáveis poderiam explicar melhor este dado. Giesen et al. ${ }^{42}$ apontam que os indivíduos com comportamento restritivo diferem seu comportamento em escolha de alimentos, se eles realizam ou não dieta. Indivíduos com comportamento restritivo que não realizam dieta tendem a "trabalhar" mais para obter "snacks", do que aqueles com comportamento restritivo e que fazem dieta. A variável "fazer dieta" parece impactar no valor reforçador do alimento. Esta variável não foi medida no presente estudo, mas pode estar relacionada com a preferência por alimentos na tarefa de escolha do software.

A influência da preocupação com a dieta e a restrição alimentar no aumento do IMC foi encontrada no estudo de Van Strien et al. ${ }^{8}$, para o sexo feminino. Os autores sugerem que este resultado parece estar relacionado com uma dificuldade maior de restrição alimentar para este público e, consequentemente, maiores episódios de compulsão alimentar, alimentação emocional e aborrecimentos diários ou ameaça social como fonte de angústia e posterior aumento do consumo calórico.

O presente estudo apresenta limitação quanto ao uso apenas do relato verbal de fome e tempo de privação dos participantes e não associação deste relato com uma medida objetiva. Sugere-se que estudos futuros 
sejam realizados utilizando medidas objetivas além do relato verbal e que seja analisado o efeito da privação e a influência das variáveis: IMC (eutrófico, magreza e excesso de peso), sexo (feminino e masculino), tempo de privação e presença de comportamento alimentar inadequado (ex: comer transtornado) na motivação para escolha de alimento e lazer (medido pelo valor reforçador).

\section{CONCLUSÃO}

A partir dos resultados encontrados neste estudo conclui-se que o tempo de privação influenciou a pontuação em alimento e lazer, em ambos os sexos, uma vez que o grupo com menor tempo de privação pontuou menos para alimento. A privação alimentar também impactou no relato de fome da amostra, observando aumento significativo no relato de fome proporcionalmente ao tempo de privação alimentar. Uma relação inversa significativa entre IMC e pontuação em alimento foi encontrada, ou seja, quanto maior o IMC dos participantes, menor a pontuação em alimentos. Embora não tenham sido exploradas neste estudo, variáveis como a presença de comportamento alimentar inadequado/comer transtornado e a prática de fazer dieta poderiam explicar esta relação.

\section{REFERÊNCIAS}

1. NDC Risk Factor Collaboration (NDC-RisC). Trends in adult body-mass index in 200 countries from 1975 to 2014: a pooled analysis of 1698 population-based measurement studies with 19,2 million participants. Lancet. 2016;387:1377-96.

2. Meldrum DR, Morris MA, Gambone JC. Obesity pandemic: causes, consequences, and solutions-but do we have the will? Fertil Steril. 2017;107(4):833-9.

3. Hales CM, Fryar CD, Carroll MD. Trends in obesity and severe obesity prevalence in US youth and adults by sex and age, 2007-2008 to 2015-2016. JAMA. 2018;319(16):1723-5.

4. Ministério da Saúde (BR). Vigitel 2016: vigilância de fatores de risco e proteção para doenças crônicas por inquérito telefônico. Brasília: Ministério da Saúde; 2016.
5. Raynor HA, Epstein LH. The relative-reinforcing value of food under differing levels of food deprivation and restriction. Appetite. 2003;40:15-24.

6. Clifton PM, Condo D, Keogh JB. Long term weight maintenance after advice to consume low carbohydrate, higher protein diets - a systematic review and meta analysis. Nutr Metabol Cardiovasc Dis. 2014;24:224-35.

7. Schaumberg K, AndersonDA, AndersonLM, Reilly EE, Gorrell S. Dietary restraint: what's the harm? A review of the relationship between dietary restraint, weight trajectory and development of eating pathology. Clin Obes. 2016;6:89-100.

8. Van Strien T, Herman CP, Verheijden MW. Dietary restraint and body mass change. A 3-year follow up study in a representative Dutch sample. Appetite. 2014;76:44-9.

9. Rolland C, Johnston KL, Lula S, Macdonald I, Broom $\mathrm{J}$. Long-therm weight loss maintenance and management following a VLCD: a 3 years outcome. Int $\mathrm{J}$ Clin Pract. 2014;68:379-87.

10. Bueno LN, Nascimento NA. Transtornos alimentares sob a perspectiva da análise do comportamento. Fragmentos de Cultura. 2014;24:37-48.

11. Neves SMM, Santos AC, Araujo L, Borges R, Quinta T, Martins W. Obesidade e a Teoria de Determinação Skinneriana: correspondências de relatos de crianças. Comportamento em foco. 2011; 1:455-68.

12. Lazzeri F. Sobre o conceito de comportamento: alguns breves comentários. ReBAC. 2013;9(2):107-12.

13. Epstein LH, Lin H, Carr KA, Fletcher KD. Food reinforcement and obesity: psychological moderators. Appetite. 2012;58(1):157-62.

14. Carr KA, Lin H, Fletcher KD, Epstein LH. Food reinforcement, dietary disinhibition and weight gain in non-obese adults. Obes. 2014;22(1):254-9.

15. Drobes DJ, Miller EJ, Hillman $\mathrm{CH}$, Bradley MM, Cuthbert BN, Lang PJ. Food deprivation and emotional reactions to food cues: implications for eating disorders. Biol Psychol. 2001;57:153-77.

16. Temple JL, Legierski CM, Giacomelli AM, Salvu SJ, 
Epstein LH. Overweight children find food more reinforcing and consume more energy than do nonoverweight children. Am J Clin Nutr.2008;87:1121-27.

17. McCullough MB, Guilkey H, Stark L. Cookie or fruit? Relative reinforcing value of snack foods among preschoolers with overweight/obesity compared to healthy weight. Appetite. 2017;111:187-94.

18. Clark EN, Dewey AM, Temple JL. Effects of daily snack food intake on food reinforcement depend on body mass index and energy density. Am J Clin Nutr. 2010;91:300-8.

19. Bobzean SA, DeNobrega AK, Perrotti LI. Sex differences in the neurobiology of drug addiction. Exp Neurol. 2014;259:64-74.

20. Dreher JC, Schmidt PJ, Kohn P, Furman D, Rubinow D, Berman KF. Menstrual cycle phase modulates reward-related neural function in women. Proc Natl Acad Sci U S A.2007;104:2465-70.

21. Lima, WF. Food or Fun (Versão 1.0) [Software]. Goiânia: Laboratório de Análise Experimental do Comportamento. 2016.

22. Catania AC. A natural science of behavior. Rev Gen Psychol. 2013;17(2):133-9.

23. Carr KA, Epstein LH. Influence of sedentary, social, and physical alternatives on food reinforcement. Health Psychol. 2018;37(2):125-31.

24. Hopkins, WG. Correlation coefficient. 2009. Disponível em: http://www.sportsci.org/resource/stats/correl.html Acessado em: 26 de junho de 2018.

25. Stice E, Burger K, Yokum S. Caloric deprivation increases responsivity of attention and reward brain regions to intake, anticipated intake, and images of palatable foods. Neuroimage. 2013;67:322-30.

26. Zitron-Emanuel N, Ganel T. The effect of food deprivation on human resolving power. Psychon Bull Rev. 2018;25(1):455-62.

27. Brace A, Yeomans MR. The reinforcing value of palatable snack foods and its relationship to subtypes of behavioural and self-report impulsivity. Eating Behaviors. 2016;21:18-23.
28. Carr KA, Lin H, Fletcher KD, Epstein LH. Food reinforcement, dietary disinhibition and weight gain in non-obese adults. Obesity. 2014;22(1):254-9.

29. Stojek MMK, MacKillop J. Relative reinforcing value of food and delay reward discounting in obesity and disordered eating: a systematic review. Clin Psychol Rev. 2017;55:1-11.

30. Epstein LH, Temple JL, Neaderhiser BJ, Salis RJ, Erbe $\mathrm{RW}$, Leddy JJ. Food reinforcement, the dopamine $\mathrm{D}_{2}$ receptor genotype, and energy intake in obese and nonobese humans. Behavioral Neuroscience. 2007;121(5):877-86.

31. Santos AFS, Souza CSV, Oliveira LS, Freitas MFL. Influência da dopamina e seus receptores na gênese da obesidade: revisão sistemática. Rev Neurocienc. 2014;22(3):373-80.

32. Carr KD, Kim G, Cabeza de vaca S. Rewarding and locomotor-activating effects of direct dopamine receptor agonists are augmented by chronic food restriction in rats. Psychopharmacology. 2001;154:420-8.

33. Caravaggio F, Raitsin S, Gerretsen P, Nakajima S, Wilson A, Graff-Guerrero A. Ventral striatum binding of a dopamine $\mathrm{D} 2 / 3$ receptor agonist but not antagonist predicts normal body mass index. Biol Psychiatry. 2015;77(2):196-202.

34. Jimenez JJ. Leisure activities and hobbies preferred by third year college students. Asia Pacific Journal of Multidisciplinary Research. 2017;5(2):21-5.

35. Cameron JD, Goldfield GS, Riou ME, Finlayson GS, Blundell JE, Doucer E. Energy depletion by diet or aerobic exercise alone: Impact of energy déficit modality on appetite parameters. Am J Clin Nutr. 2016;103(4):1008-116.

36. Derous D, Mitchell SE, Green CL, Chen L, Han JJ, Wang Y, et al. The effects of graded levels of calorie restriction: VI. Impact of short-term graded calorie restriction on transcriptomic responses of the hypothalamic hunger and circadian signaling pathways. Aging. 2016;8(4):642-61.

37. Martens MJ, Born JM, Lemmens SG, Karhunen L, Heinecke A, Goebel R, Adam TC, Westerterp-Plantenga MS. Increased sensitivity to food cues in the fasted 
state and decreased inhibitory control in the satiated state in the overweight. Am J Clin Nutr. 2013;97:4719.

38. Schoolet JW. Re-representing consciousness: Dissociations between consciousness and meta-consciousness. Trends Cogn Sci. 2002;6:339-44.

39. Perez W. Explicações comportamentais da correspondência dizer-fazer. ReBAC. 2017;13(1):16-28.

40. Creswell KG, Sayette MA, Schooler JW, Wright AGC, Pacilio LE. Visceral states call for visceral measures: verbal overshadowing of hunger ratings across assessment modalities. Assessment. 2016;25(2):173-82.

41. Saper CB, Chou TC, Elmquist JK. The need to feed: Homeostatic and hedonic control of eating. Neuron. 2002;36(2):199-211.

42. Giesen JCAH, Havermans RC, Douven A, Takelenburg M, Jansen A. Will work for snack food: the association of BMI and snack reinforcement. Obesity. 2010;18(5):966-70.

Recebido em: 12/05/2018

Aceito em: 08/08/2018 